\title{
On the Existence of Closed Orbits for a Differential System
}

\author{
Wang Hui-Feng and Yu Shu-Xiang
}

\begin{abstract}
Some criteria which verify the existence or non-existence of closed orbits for a non-linear differential system are given.
\end{abstract}

Keywords: Closed orbits, limit cycles, Poincaré-Bendixson theory

AMS subject classification: $34 \mathrm{C}$

\section{Introduction}

In this paper, we consider the existence of closed orbits for the differential system

$$
\begin{aligned}
& x^{\prime}(t)=y \\
& y^{\prime}(t)=x+x^{2}-\left(\varepsilon_{1}+\varepsilon_{2} x\right) y
\end{aligned}
$$

where $\varepsilon_{1}$ and $\varepsilon_{2}$ are real constants. It is easy to see that this system has two singular points: $O(0,0)$ (saddle point) and $A(-1,0)$ (focus or node). In [1: p: 215] the following result has been obtained:

1. If $\varepsilon_{1} \varepsilon_{2} \leq 0$, then there are no closed orbits in the system (1.1).

2. If $\varepsilon_{1} \varepsilon_{2}>0$ and $\varepsilon_{1} / \varepsilon_{2} \geq 3 / 2$, then there are again no closed orbits in the system (1.1).

3. If $\varepsilon_{1} \varepsilon_{2}>0$ and $\varepsilon_{1} / \varepsilon_{2}<3 / 2$, then the existence of closed orbits of the system (1.1) remains an open question.

In [2], the following theorem has been given.

Theorem [2: p. 94]: If $\varepsilon_{1}=\varepsilon_{2}$, then there exists a neighbourhood of the point $(-1,0)$, such that there are infinitely many limit cycles within this neighbourhood with $(-1,0)$ in their interiors.

The system (1.1) is a special form of quadratic differential systems. A considerable number of papers have been written in connection with limit cycles of quadratic systems (see, e.c., [3]).

Wang Hui-Feng: Hainan Teachers Coll., Dep. Math., 571158 Hainan Province, China Yu Shu-Xiang: Acad. Sinica, Inst. Math., 100080 Beijing, China 


\section{The main results}

Below $f^{\prime}$ for a function $f=f(t)(t \in \mathbb{R})$ always mean the derivation for $t$. In this paper, we shall prove the following theorems.

Theorem 2.1: Suppose $\varepsilon_{1} \varepsilon_{2}>0$. If $\varepsilon_{1} \leq \varepsilon_{2}-2$ or $\varepsilon_{1} \geq \varepsilon_{2}+2$, then there are no closed orbits in the system (1.1).

Proof: Setting

$$
F(x, y)=y-k(x+1)=0
$$

we have

$$
\begin{aligned}
\left.F^{\prime}\right|_{(|\cdot|)}=y^{\prime}-k x^{\prime} & =x+x^{2}-\left(\varepsilon_{1}+\varepsilon_{2} x\right) y-k y \\
& =\left(1-k \varepsilon_{2}\right) x^{2}+\left(1-k \varepsilon_{1}-k \varepsilon_{2}-k^{2}\right) x-k \varepsilon_{1}-k^{2}
\end{aligned}
$$

Consider the equation

$$
k^{2}-k\left(\varepsilon_{2}-\varepsilon_{1}\right)+1=0
$$

Clearly, if

$$
\left(\varepsilon_{2}-\varepsilon_{1}\right)^{2}-4 \geq 0
$$

then there is one real root $k_{1}$ which is a solution of the equation (2.3). When $k=k_{1}$; from (2.2) we obtain

$$
\left.F^{\prime}\right|_{(|\cdot|)}=\left(1-\varepsilon_{2} k_{1}\right)(x+1)^{2} .
$$

It is easy to see that $1-\varepsilon_{2} k_{1} \neq 0$ when $\varepsilon_{1} \varepsilon_{2}>0$. Therefore the straight line $y=k_{1}(x+1)$ is a line without contact, i.e., the trajectory passing through any point $P$ on the line (except for the point $A$ ) must cross the line in the same sense. Thus there are no closed orbits around the popint $A$ in the system (1.1)

Theorem 2.2: Suppose $\varepsilon_{1} \varepsilon_{2}>0$. If $\varepsilon_{1}=\varepsilon_{2}$, then there are. no closed orbits and singular closed orbits in the system (1.1).

Proof: When $\varepsilon_{1}=\varepsilon_{2} \neq 0$, the system (1.1) can be written as

$$
\begin{aligned}
x^{\prime}=y & \equiv P(x, y) \\
y^{\prime}=x+x^{2}-\varepsilon_{2}(x+1) y & \equiv Q(x, y)
\end{aligned}
$$

Consider the function which is similar to that in [3: Section 12]

$$
B(x, y)=(y-\alpha x)^{-\alpha \varepsilon_{2}} \exp \left(-\alpha \varepsilon_{2}(x+1)\right)
$$

where $\alpha>0$ is a constant that will be defined below. We have

$$
\begin{aligned}
\frac{\partial}{\partial x}(B P)+\frac{\partial}{\partial y}(B Q)= & (y-\alpha x)^{-\alpha \varepsilon_{2}-1} \\
& \times\left(-\alpha \varepsilon_{2} y^{2}+\left(\alpha^{2} \varepsilon_{2}+\alpha \varepsilon_{2}^{2}-\varepsilon_{2}\right) y(x+1)\right) \\
& \times \exp \left(-\alpha \varepsilon_{2}(x+1)\right) .
\end{aligned}
$$


Set

$$
\alpha^{2}+\varepsilon_{2} \alpha-1=0
$$

Let $\alpha_{1}>0$ be a root of the equation (2.8). Then, from (2.7) we obtain

$$
\frac{\partial}{\partial x}(B P)+\frac{\partial}{\partial y}(B Q)=\left(y-\alpha_{1} x\right)^{-\alpha_{1} \varepsilon_{2}-1}\left(-\alpha_{1} \varepsilon_{2} y^{2}\right) \exp \left(-\dot{\alpha}_{1} \varepsilon_{2}(x+1)\right)
$$

Obviously, the straight line

$$
\Phi(\dot{x}, y) \equiv y-\alpha_{1} x=0
$$

passes through two points $(0,0)$ and $\left(-1,-\alpha_{1}\right)$ in the $(x, y)$-plane, and we have

$$
\left.\Phi^{\prime}\right|_{(1.1)}=y^{\prime}-\alpha_{1} x^{\prime}=x+x^{2}-\varepsilon_{2}(x+1) y-\alpha_{1} y=\left(1-\alpha_{1} \varepsilon_{2}\right) x^{2} .
$$

Therefore the straight line $\dot{y}=\alpha_{1} \dot{x}$ is a line without contact, i.e., the trajectory passing through any point $P$ on the line (except for the point $O$ ) must cross the line in the same sense. Thus each closed orbit can not intersect the line $\Phi=0$. Moreover, the function $\frac{\partial(B P)}{\partial x}+\frac{\partial(B Q)}{\partial y}$ does not change sign in the region $y-\alpha_{1} x>0$, and it does not vanish identically in any subregion of this region. By Dulac's theorem (see [1: $p$. 205]) it follows that there are no closed orbits and singular closed orbits in the region $y-\alpha_{1} x>0$. Note that the singular point $A(-1,0)$ lies in the region $y-\alpha_{1} x>0$, thus there are no closed orbits of the system $(1.1)$ in the $(x, y)$-plane

Remark: A singular closed orbit means a simple closed curve which is the union of alternating non-closed whole orbits and singular points (see [1: p. 205]).

Theorem 2.3: Suppose $\varepsilon_{1} \varepsilon_{2}>0$. If $\varepsilon_{2}\left(\varepsilon_{1}-\varepsilon_{2}\right)>0$, then there are no closed orbits in the system (1.1).

Proof: For convenience, we set $x=x_{1}-1$ and $y=y_{1}$. By (1.1), we get the system

$$
\begin{aligned}
& x_{1}^{\prime}=y_{1} \\
& y_{1}^{\prime}=-x_{1}+\left(\varepsilon_{2}-\varepsilon_{1}\right) y_{1}+x_{1}^{2}-\varepsilon_{2} x_{1} y_{1} .
\end{aligned}
$$

Obviously, the system (2.12) in the $\left(x_{1}, y_{1}\right)$-plane has two singular points: $O_{1}(0,0)$ (focus or node) and $A_{1}(1,0)$ (saddle point). When $\varepsilon_{1}=\varepsilon_{2}$, then the system (2.12) can be written as

$$
\begin{aligned}
& x_{1}^{\prime}=y_{1} \\
& y_{1}^{\prime}=-x_{1}+x_{1}^{2}-\varepsilon_{2} x_{1} y_{1} .
\end{aligned}
$$

At first we prove that the singular point $O_{1}$ is a focus of the system (2.13). We use a classical method (see [4: p. 180]). Set $x_{1}=r \cos \theta$ and $y_{1}=r \sin \theta$. Then (2.13) yields

$$
\begin{aligned}
& r^{\prime}=r^{2} \sin \theta \cdot\left(\cos ^{2} \theta-\varepsilon_{2} \sin \theta \cos \theta\right) \\
& \theta^{\prime}=-1+r \cos \theta \cdot\left(\cos ^{2} \theta-\varepsilon_{2} \sin \theta \cos \theta\right) .
\end{aligned}
$$

Hence we obtain by division the relation

$$
\frac{d r}{d \theta}=\frac{r^{2} \sin \theta \cdot\left(\cos ^{2} \theta-\varepsilon_{2} \sin \theta \cos \theta\right)}{-1+r \cos \theta \cdot\left(\cos ^{2} \theta-\varepsilon_{2} \sin \theta \cos \theta\right)} \equiv R(r, \theta)
$$


and

$$
R(r, \theta)=r^{2} R_{2}(\theta)+r^{3} R_{3}(\theta)+\ldots+r^{n} R_{n}(\theta)+\ldots
$$

where the series converges for $r$ sufficiently small and any $\theta$. In fact, we have

$$
R_{n}(\theta)=R_{n-1}(\theta) \cos \theta \cdot\left(\cos ^{2} \theta-\varepsilon_{2} \sin \theta \cos \theta\right) \quad\left(n \geq 3 ; R_{1}=-\sin \theta\right) .
$$

Let

$$
r=f\left(\theta, r_{0}\right)=u_{1}(\theta) r_{0}+u_{2}(\theta) r_{0}^{2}+\ldots
$$

be the solution of relation (2.14) satisfying $f\left(\theta, r_{0}\right)=r_{0}$. Then we must have

$$
u_{1}(0)=1 \quad \text { and } \quad u_{n}(0)=0 \quad(n \geq 2) .
$$

By substituting (2.15) into (2.14) and identifying equal powers of $r$, we thus obtain a system

$$
\begin{aligned}
& u_{1}^{\prime}=0 \\
& u_{2}^{\prime}=R_{2}(\theta) u_{1}^{2}=R_{2}(\theta) \\
& u_{3}^{\prime}=2 u_{1} u_{2} R_{2}(\theta)+u_{1} R_{3}(\theta)
\end{aligned}
$$

By (2.17) and (2.16), we get

$$
\begin{aligned}
u_{1}(\theta)= & 1 \\
u_{2}(\theta)= & \frac{1}{3}\left(-1+\cos ^{3} \theta+\varepsilon_{2} \sin ^{3} \theta\right) \\
u_{3}(\theta)= & \frac{5}{18}\left(\cos ^{6} \theta-1\right)+\frac{1}{9} \varepsilon_{2}^{2} \sin ^{6} \theta+\frac{2}{9}\left(\cos ^{3} \theta-1\right)-\frac{2}{9} \varepsilon_{2} \sin ^{3} \theta \\
& +\frac{1}{16} \varepsilon_{2}^{2}(\cos 2 \theta-1)-\frac{1}{48} \varepsilon_{2}^{2}\left(\cos ^{3} 2 \theta-1\right) \\
& -\frac{1}{3} \varepsilon_{2}\left(\theta+\frac{1}{2} \sin 2 \theta\right)+\varepsilon_{2}\left(\frac{3}{2} \theta+\sin 2 \theta+\frac{1}{8} \sin 4 \theta\right) \\
& -\frac{10}{3} \varepsilon_{2}\left(\frac{5}{16} \theta+\frac{15}{64} \sin 2 \theta+\frac{3}{64} \sin 4 \theta+\frac{1}{192} \sin 6 \theta\right)
\end{aligned}
$$

hence

$$
u_{1}(2 \pi)=1, \quad u_{2}(2 \pi)=0, \quad u_{3}(2 \pi)=\frac{\pi}{4} \varepsilon_{2} .
$$

Therefore, from (2.15) we obtain.

$$
r(2 \pi)-r_{0}=\frac{\pi}{4} \varepsilon_{2} r_{0}^{3}\left(1+\mu_{1} r_{0}+\mu_{2} r_{0}^{2}+\ldots\right)
$$

By this and the fact that $\theta^{\prime}<0$ for $r$ sufficiently small and any $\theta$, we know that when $\varepsilon_{2}>0$, then the singular point $O_{1}$ is a stable focus, and when $\varepsilon_{2}<0$, then $O_{1}$ is an unstable focus. From (2.12) we obtain by division the relation

$$
\frac{d y_{1}}{d x_{1}}=\frac{-x_{1}+x_{1}^{2}-\varepsilon_{2} x_{1} y_{1}}{y_{1}}+\left(\varepsilon_{2}-\varepsilon_{1}\right)
$$


i.e.

$$
\left.\frac{d y_{1}}{d x_{1}}\right|_{(2.12)}=\left.\frac{d y_{1}}{d x_{1}}\right|_{(2.13)}+\left(\varepsilon_{2}-\varepsilon_{1}\right)
$$

for $y_{1} \neq 0$.

At first, we assume that $\varepsilon_{2}<0$ and $\varepsilon_{2}-\varepsilon_{1}>0$. Then $O_{1}$ is an unstable focus of the system (2.13) and $O_{1}$ is also an unstable focus of the system (2.12) (by Theorem 2.1 we can consider the case $\left(\varepsilon_{2}-\varepsilon_{1}\right)^{2}-4<0$ only). Moreover, it follows from Theorem 2.2 that the system (2.13) has no closed orbits and singular closed orbits.

We need to prove that the system (2.12) has no closed orbits. The following proof proceeds by reduction to absurdity. Suppose the system (2.12) has one closed orbit $\Gamma$. Let $G$ be the region bounded by $\Gamma$ such that $O_{1} \in G$. Let the closed orbit $\Gamma$ intersect the half axes $y_{1}>0, x_{1}>0, y_{1}<0$ and $x_{1}<0$ at $M, N, P$ and $Q$, respectively. Clearly, the point $N$ must lie between $O_{1}$ and $A_{1}$. Let $\gamma^{+}(M)$ be the positive semi-trajectory of the system (2.13), originating from $M$. It follows from(2.20) that $\gamma^{+}(M)$ must lie in $G$ for $t>0$ sufficiently small. We claim that $\gamma^{+}(M)$ lies in $G$ for all $t>0$. If not, there is $t=t_{1}>0$ such that $\gamma^{+}(M)$ meets $\Gamma$ at $N$ or $Q$ for $t=t_{1}$ (since the relation (2.20) implies that $\gamma^{+}(M)$ can not meet $\Gamma$ at other points for $\left.t>0\right)$. Let $\gamma^{+}(M)$ meet $\Gamma$ at $N$. Then, in the closed region $H=\left\{\left(x_{1}, y_{1}\right) \in \mathbb{R}^{2}: x_{1}, y_{1} \geq 0\right\}$, the curvilinear figure $M N M$ made up of the trajectory arc $M N$ of $\Gamma$, and the trajectory arc $M N$ of $\gamma^{+}(M)$ encloses a region, denoted by $K(\subset G)$. The positive semi-trajectory of the system (2.13) originating from any point on the open trajectory arc $M N$ of $\Gamma$ must enter $K$ for $t>0$ and stays in $K$ for all $t>0$. By the Poincaré-Bendixson theory of planar systems, it follows that there is at least one critical point in $K$. This is impossible because the system (2.13) has the singular points $O_{1}$ and $A_{1}$ only. Similarly, we can prove that $\gamma^{+}(M)$ cannot meet $\Gamma$ at $Q$. Moreover, it follows from Theorem 2.2 that the system (2.13) has no closed orbits. Thus $\gamma^{+}(M)$ cannot return to $M$ for $t>0$, and must tend to the singular point $O_{1}$ as $t \rightarrow+\infty$. But this contradicts the fact that $O_{1}$ is an unstable focus of the system (2.13) when $\varepsilon_{2}<0$. Therefore, the system (2.12) has no closed orbits.

In the case that $\varepsilon_{2}>0$ and $\varepsilon_{2}-\varepsilon_{1}<0$, the proof is similar. Instead of $\gamma^{+}(M)$, we consider the negative semi-trajectory $\gamma^{-}(M)$ of the system (2.13) originating from $M$, and we can prove that $\gamma^{-}(M)$ stays in $G$ for all $t<0$ (note that (2.20) implies $\left.\frac{d y_{1}}{d x_{1}}\right|_{(2.12)}<\left.\frac{d y_{1}}{d x_{1}}\right|_{(2.13)}$ for $y_{1} \neq 0$ and $\left.\varepsilon_{2}-\varepsilon_{1}<0\right)$. This contradicts the fact that $O_{1}$ is a stable focus of the system (2.13) when $\varepsilon_{2}>0$. Therefore, the system (2.12) also has no closed orbits in this case

Theorem 2.4: Suppose $\varepsilon_{1} \varepsilon_{2}>0$. If $\varepsilon_{2}\left(\varepsilon_{1}-\varepsilon_{2}\right)<0$ and $\left|\varepsilon_{1}-\varepsilon_{2}\right|$ is sufficiently small, then there are closed orbits of the system (1.1) in some neighbourhood of the singular point $O$.

Proof: We set $\varepsilon_{2}-\varepsilon_{1}=\lambda$ and write the system (2.12) in the form

$$
\begin{aligned}
& x_{1}^{\prime}=y_{1} \\
& y_{1}^{\prime}=-x_{1}+\lambda y_{1}+x_{1}^{2}-\left(\lambda+\varepsilon_{1}\right) x_{1} y_{1} .
\end{aligned}
$$

If $\lambda=0$, we obtain from $(2: 12)_{\lambda}$ the system (2.13) (note $\varepsilon_{1}=\varepsilon_{2}$ ).

First, we assume that $\varepsilon_{2}>0$. Then, as proved above, the singular point $O_{1}$ is a stable focus of the system (2.13). Moreover, it is easy to see that if $\lambda>0$ and sufficiently 
small, then $O_{1}$ is an unstable focus of the system (2.12) $)_{\lambda}$. Therefore (see [5: Section 25.3]), there are a sufficiently small neighbourhood $V$ of the point $O_{1}$ and a small $\delta_{0}>0$ such that any system (2.12) ${ }_{\lambda}$ for which $\lambda<\delta_{0}$ has a unique closed orbit in $V$.

Similarly, if $\varepsilon_{2}<0$, then $O_{1}$ is an unstable focus of the system (2.13). And, when $\lambda<0$ and sufficiently small, then $O_{1}$ is a stable focus of the system (2.12) $\lambda$. Therefore, there are a sufficiently small neighbourhood $W$ of $O_{1}$ and a small $\delta_{1}>0$ such that any system (2.12) त $_{\lambda}$ for which $|\lambda|<\delta_{1}$ with $\lambda<0$ has a unique closed orbit in $W$ tems.

Remark that also the system $(2.12)_{\lambda}$ is a special form of quadratic differential sys-

\section{References}

[1] Andronov, A.A., Leontovich, E.A., Gordon, I.I. and A.G. Maier: Qualitative Theory of Second-Order Dynamic Systems. New York: Wiley 1973.

[2] C. Hsin: A note on the existence of periodic solutions of a differential system. Lect. Notes Math. 1342 (1988), 94 - 99.

[3] Y. Y.-Q. (ed.): Theory of Limit Cycles (Trans. Math. Monogr.: Vol. 66). Providence (R.I.): Amer. Math. Soc. 1986.

[4] Lefschetz, S.: Differential Equations: Geometric Theory. New York: Intersci. 1957.

[5] Andronov, A.A., Leontovich, E.A., Gordon, I.I. and A.G. Maier: Theory of Bifurcations of Dynamic Systems on a Plane. New York: Wiley 1973.

Received 10.11.1993 